\title{
Kompressionstherapie nach operativer Varizensanierung
}

\author{
B. Cucuruz; J. Noppeney; M. Bourdy; A. Korte; T. Noppeney \\ MVZ Obere Turnstraße, Nürnberg
}

\begin{abstract}
Schlüsselwörter
Postoperative Kompressionstherapie, Varikose, Crossektomie, Radiofrequenzablation
\end{abstract}

\section{Zusammenfassung}

Einleitung: Die Kompressionstherapie ist bis heute eine Alternative zur operativen Varizensanierung und der Goldstandard in der Therapie chronisch venöser Insuffizienz. Umstritten ist die Frage der postoperativen Kompressionstherapie. Ziel dieser Arbeit ist es einen Überblick der aktuellen Literatur bezüglich der postoperativen Kompressionstherapie zu bieten.

Methodik: Es wurde eine Literaturrecherche in Medline, pubmed und Embase mit dem Thema der operative Varizensanierung durchgeführt. Aus den Arbeiten wurde die jeweils verwendete postoperative Kompressionstherapie erfasst.

Ergebnisse: Es wurden 182 Studien erfasst, davon jedoch nur 7 randomisierte Studien Die Kompressionstherapie variiert von keiner bis zu 6 Wochen langen Kompression. Es wurde immer eine elastische Kompression verwendet, zumeist Kompressionsstrümpfe.

Schlussfolgerung: Die postoperative Kompressionstherapie nach operativer Varizensanierung wird unabhängig der verwendeten operativen Methode empfohlen. Aufgrund der eingeschränkten Datenlage gibt es keine Empfehlung zur Dauer der postoperativen Kompression oder eine empfohlene Kompressionsstrumpflänge.

\section{Keywords}

Compression therapy, varicose vein treatment, radiofrequency

\section{Summary}

Introduction: Compression therapy has always been the gold standard of chronic vein insufficiency and an alternative to operative treatment of varicose veins. Postoperative compression therapy on the other hand stays controversial. The purpose of this work was to give a summary of the existing literature of compression therapy after operative varicose vein treatment

Methods: A literature research in Medline, Pubmed and Embase was performed. Publications about operative varicose vein treatment were used to assess the used postoperative compression therapy.

Results: There were 182 studies about operative varicose vein treatment found, but only 7 randomized studies. The compression therapy varies from no compression to a compression therapy of 6 weeks with elastic bandages or compression stockings.

Conclusion: Postoperative compression therapy after varicose vein surgery despite of what operation method is used, is always recommended. There is no recommendation to the duration of postoperative compression therapy.

Korrespondenzadresse

Dr. Thomas und Dr. Jeanette Noppeney

MVZ Obere Turnstraße

Obere Turnstraße 8-10, 90429 Nürnberg

E-Mail: tn@gefaesszentrum-nuernberg.de
Phlebologie 2018;47: 17-29

https;//doi.org/10.12687/phleb2407-1-2018

Eingereicht: 18. Dezember 2017

Angenommen: 18. Dezember 2017

English version available at:

www.phlebologieonline.de
Die Kompression ist bereits seit dem Altertum das Therapieverfahren der Wahl für venöse Erkrankungen der unteren Extremität (1-3). Für eine chronisch venöse Insuffizienz bleibt die Kompressionstherapie das Mittel der Wahl. Für die oberflächliche Varikose gibt es neben der klassischen offenen Crossektomie mit Stripping der Vena saphena magna zahlreiche endovenöse Verfahren: verschiedene Sklerosierungs-Verfahren, Radiofrequenzablation, Laserablation und Cyanocrylat. Durch Kompressionstherapie werden die Nebeneffekte der chirurgischen Sanierung reduziert ( $\$$ Tab. 1).

Bereits 1979 konnte mittels randomisierter Studien eine signifikante Reduktion der Schmerzen, des stationären Aufenthaltes und eine verbesserte Wundheilung durch postoperative Kompression erzielt werden (4). Mittels radiomarkierter Erythrozyten wurde an 10 Probanden mit bilateraler Crossektomie und Stripping der Vena saphena magna, bei welchen ein Bein postoperativ elastisch mit $40 \mathrm{mmHg}$ gewickelt (Testbein) wurde, das andere nur mit $10-20 \mathrm{mmHg}$ (Kontrollbein), eine signifikante Reduktion des subkutanen Hämatoms im Testbein festgestellt (5).

Houtermans-Auckel zeigte mittels photoelektrischer Volumenmessung des Beines an 104 Patienten vor und nach Varizenstripping eine signifikante Reduktion des Beinumfangs durch Kompressionsstrümpfe (6). Die Beinvolumenmessung wurde präoperativ, 3 Tage, 2 Wochen und 4 Wochen postoperativ durchgeführt. Alle Probanden erhielten für mindestens 3 Tage postoperativ eine elastokompressive Wickelung des Beins. Die Kontrollgruppe wies bereits bei der ersten Messung 3 Tage postoperativ eine Reduktion des Beinvolums auf. Die Kontrollgruppe konnte signifikant früher ihre Arbeit aufnehmen (nach durchschnittlich 11 Tagen), im Vergleich zur Testgruppe (durchschnittlich 15 Tage). Eine weitere Nachkontrolle nach Beendigung der 4 Wochen wurde nicht durchgeführt. 
Tab. 1 Postoperative Nebeneffekte, welche durch Kompression reduziert werden können

\begin{tabular}{|c|c|}
\hline \multicolumn{2}{|c|}{$\begin{array}{l}\text { Operative Varizensanierung (offen oder } \\
\text { endovenös) }\end{array}$} \\
\hline $\begin{array}{l}\text { Durch Kompression } \\
\text { reduzierte postopera- } \\
\text { tive Nebeneffekte }\end{array}$ & $\begin{array}{l}\text { - Hämatom } \\
\text { - Ödem } \\
\text { - Schmerzen } \\
\text { - Thrombose } \\
\text { - Thrombophlebitis } \\
\text { - Hyperpigmentierung }\end{array}$ \\
\hline
\end{tabular}

In den aktuellen europäischen Leitlinien wird eine Kompressionstherapie nach operativer Varizensanierung, unabhängig von der Methode, empfohlen (Evidenzkathegorie A, Empfehlungsklasse I, 7). Die postoperative Kompressionstherapie wird in Frankreich von $97,1 \%$ nach klassischer Varizenchirurgie angewendet (8).

In der aktuellen Literatur findet man 182 Studien, welche sich mit der Kompressionstherapie nach operativer Varizenchirurgie befassen, allerdings nur 7 randomisierte Studien ( Tab. 2, 9). Die Kompressionstherapie variiert von keiner bis zu 6 Wochen langen Kompression. Es wird immer eine elastische Kompression verwendet, zumeist Kompressionsstrümpfe. Nach offener Varizensanierung wird einheitlich eine Kompressionstherapie von mindestens 3 Tagen durchgeführt.

Nach Laserablation kommt es durch eine Kompressionstherapie von 7-14 Tagen zu einer signifikanten Schmerzreduktion und erhöhtem Wohlbefinden $(14,15)$ $(\checkmark$ Tab. 2). Die Studien sind sehr heterogen, es gibt keine postoperative Anleitung der Patienten. In allen Studien sind nur vereinzelt Patienten mit einem varikösen Befund an beiden Beinen. Patienten mit Rezidivvarikosis wurden von Elderman et al. ausgeschlossen (15). Bei den vorliegenden
Studien mit endovenösen Verfahren, wurden keine Mini-Phlebektomien durchgeführt. Dementsprechend sind sie schonender, und man konnte auf eine Kompressionstherapie verzichten (12). Im klinischen Alltag wird jedoch meistens zur Entfernung der Seitenäste zusätzlich zu dem endovenösen Verfahren auch eine Miniphlebektomie durchgeführt, welche ein postoperatives Hämatom und ein Ödem zur Folge hat. Zudem unterstützt eine Kompressionstherapie nach endovenösen Verfahren den Rückgang der Stammvene $(7,13)$.

Dieses uneinheitliche Vorgehen der Operateure unterstreicht die Arbeitsgruppe von El-Sheikha. Mittels Fragebogen wurden 348 Operateure aus Großbritannien und Irland befragt. Gefragt wurde nach der operativen Methode, Art und Dauer der Kompression nach Schaumsklerosierung, Art und Dauer der Kompression nach endovenöser Laser- oder Radiofrequenzablation, Art der postoperativen Analgesie. Das Ergebnis war sehr heterogen mit einer Kompressionsdauer von 0-96 Tagen. Ebenso war die verwendete Art der Kompression sehr uneinheitlich: Kompressionsstrümpfe, Wickelung, Auflagen, und verschiedene Kombinationen der vorhandenen Möglichkeiten (10). Aufgrund dieser eingeschränkten Datenlage gibt es in der europäischen Leitlinie keine Empfehlung zur Dauer der postoperativen Kompression. Ebenso wenig wird die Höhe der Kompression (nur Unterschenkelkompression oder doch gesamtes Bein) empfohlen (7).

\section{Diskussion}

Die Reduktion von postoperativen subkutanen Hämatomen durch Kompression konnte gezeigt werden (5). Allerdings wur- de dies an einem Kollektiv von 10 Patienten untersucht. Eine Reduktion des Beinödems durch Kompression wurde mittels Umfangmessung 3 Tage, 2 und 4 Wochen nach operativen Varizensanierung gezeigt (6). Allerdings gibt es hier keine Langzeitdaten zu diesen Patienten. Gleicht sich das Beinvolumen nach Absetzten der Kompression wieder dem Ausgangswert an? Nach wie viel Zeit? Wie sehr wirkt sich eine Sitzende Tätigkeit auf das Beinvolumen aus? Als Argument gegen die Kompressionstherapie bringt die gleiche Studie, dass Patienten ohne Kompressionstherapie signifikant früher ihre Arbeit aufgenommen haben (6).

Die neuen endovenösen Verfahren sind schonender. In den vorliegenden Studien wurde bei den endovenösen Verfahren keine zusätzliche Phlebektomie durchgeführt $(6,10$ - 15). Um hier eine Schmerzreduktion nach Miniphlebektomie zur erreichen ist eine Kompressionstherapie hilfreich. Zudem unterstützt eine Kompressionstherapie nach endovenösen Verfahren den Rückgang der Stammvene $(7,13)$. Aus den bisherigen Studien, meint man, dass eine postoperative Kompression von 7 Tagen hilfreich ist. Aufgrund des uneinheitlichen Protokolls mit unterschiedlichen Arten der postoperativen Kompression, postoperatives Management und Aufklärung des $\mathrm{Pa}-$ tienten gibt es weder zur Länge noch zur Dauer der Kompression eine Empfehlung der Leitlinie (7).

Neue Studien, mit einheitlichem Protokoll sind dringend von Nöten um zum einen die Evidenz der postoperativen Kompression zu belegen und eine Vereinheitlichung der postoperativen Kompressionstherapie zu erreichen.

\begin{tabular}{|l|l|l|l|l|l|l}
\hline Autor & Jahr & Patienten & Therapie & Kompressionsart & Tage (d) & Beurteilung \\
\hline El-Sheikha (10) & 2016 & $348^{*}$ & endovenös & MTS/ Wickelung & 7,5 (2-30) & uneinheitliches Regime \\
\hline Biswas (11) & 2007 & 110 & offen & MTS/ Wickelung & 7 oder 21 & kein Unterschied \\
\hline Houtermans-Auckel JP (6) & 2009 & 104 & offen & MTS/ Wickelung & 3 oder 28 & kein Unterschied \\
\hline Hamel-Desnos CM (12) & 2010 & 60 & endovenös & MTS & 0 oder 21 & kein Unterschied \\
\hline O’Hare JL (13) & 2010 & 124 & endovenös & Wickelung & 1 oder 5 & kein Unterschied \\
\hline Bakker NA (14) & 2013 & 109 & endovenös & MTS & 2 oder 7 & Kompression für 7 Tage \\
\hline Elderman JH (15) & 2014 & 111 & endovenös & Wickelung & 1 oder 14 & Kompression für 14 Tage \\
\hline
\end{tabular}

Tab. 2

Literaturübersicht (adaptiert nach 9); *348 Ärzte wurden über die verordnete Kompression befragt 


\section{Schlussfolgerung}

Die postoperative Kompressionstherapie nach operativer Varizensanierung wird unabhängig der verwendeten operativen Methode in den europäischen Leitlinien empfohlen (7, Evidenzkategorie A, Empfehlungsklasse I). Aufgrund der eingeschränkten Datenlage gibt es keine Empfehlung zur Dauer der postoperativen Kompression oder eine empfohlene Kompressionsstrumpflänge.

\section{Interessenkonflikt}

Dr. Thomas Noppeney, Beratervertrag mit Firma Medi GmbH, Bayreuth

\section{Ethische Richtlinien}

Für das Manuskript wurden keine Studien an Menschen oder Tieren durchgeführt.

\section{Literatur}

1. Carradice D. Superficial venous insufficiency from the infernal to the endothermal. Ann R Coll Surg Engl 2014; 96: 5-10.
2. Royle J and Somjen GM. Varicose veins: Hippocrates to Jerry Moore. ANZ J Surg 2007; 77: $1120-1127$

3. Pocard M. Varicose veins and methods used to cut them:from the Ebers papyrus to Trendelenburg. Ann Chir 1997; 51: 710-712.

4. Melrose DG, Knight MT, Simandl E The stripping of varicose veins: a clinical trial of intermittent compression dressings. Br J Surg 1979; 66: 53-55.

5. Travers JP, Rhodes JE. Postoperative limb compression in reduction of haemorrhage after varicose vein surgery. Ann R Coll Surg Engl 1993; 75: 119-122.

6. Houtermans-Auckel JP, van Rossum E, Teijink JA, Dahlmans AA, Eussen EF, Nicolaï SP, Welten RJ. To wear or not to wear compression stockings after varicose vein stripping: a randomised controlled trial. Eur J Vasc Endovasc Surg 2009 Sep; 38(3): 387-391.

7. Writing Committee, Wittens C, Davies AH, Baekgaard N, Broholm R, Cavezzi, A, Chastanet S, et al. Editor's Choice - Management of Chronic Venous Disease. Clinical Practice Guidelines of the European Society for Vascular Surgery (ESVS). Eur J Vasc Surg (2015) 49, 678-737.

8. Rastel D Perrin M, Guidicelli M. Compressive therapy after varicose vein surgery: results of a French national inquiry. J Mal Vasc 2004; 29: 27-34.

9. J. El-Sheikha, D. Carradice, S. Nandhra, C. Leung, G.E. Smith, B. Campbell and I.C. Chetter. Systematic review of compression following treatment for varicose veins. J Vasc Nurs. 2016 Mar;34(1):31

10. El-Sheikha J, Nandhra S, Carradice D, Acey C, Smith GE, Campbell B, Chetter IC. Compression regimes after endovenous ablation for superficial venous insufficiency--A survey of members of the Vascular Society of Great Britain and Ireland. Phlebology 2016 Feb; 31(1): 16-22

11. Biswas S, Clark A, Shields DA. Randomised clinical trial of the duration of compression therapy after varicose vein surgery. Eur J Vasc Endovasc Surg 2007 May; 33(5): 631-637.

12. Hamel-Desnos CM, Guias BJ, Desnos PR, Mesgard A. Foam sclerotherapy of the saphenous veins: randomised controlled trial with or without compression. Eur J Vasc Endovasc Surg 2010 Apr; 39(4): 500-507.

13. O'Hare JL, Stephens J, Parkin D, Earnshaw JJ. Randomized clinical trial of different bandage regimens after foam sclerotherapy for varicose veins. Br J Surg 2010 May; 97(5): 650-656.

14. Bakker NA, Schieven LW, Bruins RM, van den Berg M, Hissink RJ. Compression stockings after endovenous laser ablation of the great saphenous vein: a prospective randomized controlled trial. Eur J Vasc Endovasc Surg. 2013 Nov; 46(5): 588-592

15. Elderman JH, Krasznai AG, Voogd AC, Hulsewé KW, Sikkink CJ. Role of compression stockings after endovenous laser therapy for primary varicosis. J Vasc Surg Venous Lymphat Disord 2014 Jul; 2(3): 289-296.

16. Kern R, Ramelet AA, Wutschert R, Hayoz D. Compression after sclerotherapy for telangiectasias and reticular leg veins: a randomized controlled study. J Vasc Surg 2007; 45: 1212-1216. 